\title{
Conformal external beam radiation or selective internal radiation therapy-a comparison of treatment outcomes for hepatocellular carcinoma
}

\author{
Oluwadamilola T. Oladeru ${ }^{1}$, Joseph A. Miccio ${ }^{1}$, Jie Yang $^{2,3}$, Yaqi Xue ${ }^{3}$, Samuel Ryu ${ }^{1}$, Alexander M. Stessin ${ }^{1}$ \\ ${ }^{1}$ Department of Radiation Oncology, ${ }^{2}$ Department of Preventive Medicine, ${ }^{3}$ Department of Applied Mathematics and Statistics, Stony Brook \\ University, Stony Brook, NY 11794, USA \\ Contributions: (I) Conception and design: AM Stessin; (II) Administrative support: S Ryu, AM Stessin; (III) Provision of study materials or patients: \\ None; (IV) Collection and assembly of data: OT Oladeru, JA Miccio; (V) Data analysis and interpretation: All authors; (VI) Manuscript writing: All \\ authors; (VII) Final approval of manuscript: All authors. \\ Correspondence to: Alexander M. Stessin, MD, PhD. Department of Radiation Oncology, HSC T-3, Stony Brook University, Stony Brook, NY 11794- \\ 8165, USA. Email: Alexander.Stessin@stonybrookmedicine.edu.
}

Background: Non-operative treatment for hepatocellular carcinoma (HCC) has expanded significantly with the use of selective internal radiotherapy (SIRT) mostly with yttrium $90\left({ }^{90} \mathrm{Y}\right)$ tagged microspheres and highly conformal external beam radiation therapy such as stereotactic body radiotherapy (SBRT) to treat unresectable liver tumors for local tumor control. SBRT is a noninvasive procedure using external radiation source under image guidance, while SIRT delivers radioactive particles by transarterial radioembolization (TARE). However, the survival benefits of SBRT versus SIRT have never been compared. The aim of the present study is to compare the outcomes of overall and disease specific survival (DSS) using SIRT versus SBRT to treat HCC.

Methods: The Surveillance, Epidemiology, and End Results (SEER) registry database [2004-2011] was queried for cases of unresectable HCC. Patients with missing data and those who received surgery were excluded from the study. A total of 189 patients with unresectable HCC were identified and used for statistical analysis, with 112 receiving SBRT and 77 receiving SIRT. Overall and disease-specific survival was compared using multivariable cox proportional hazard models.

Results: After adjusting for confounding factors (age at diagnosis, gender, race, grade, stage, AFP level and type of surgery), there were no significant difference in overall survival (OS) [hazard ratio (HR), 0.72; 95\% confidence interval (CI), 0.49-1.07; P=0.1077] and DSS (HR, 0.70; 95\% CI, 0.46-1.05; P=0.0880) for SIRT compared to SBRT. However, patients with elevated AFP level were associated with higher death risk $(\mathrm{P}=0.0459)$ and disease specific death risk $(\mathrm{P}=0.0233)$ than those with AFP within normal limits in both treatment groups.

Conclusions: The retrospective analysis serves as the first comparison of SIRT to SBRT in treatment of unresectable HCC. Our findings suggest both treatment approaches result in similar outcomes in overall and disease-specific survival benefit. Future prospective randomized trials are needed to better evaluate and compare the two radiation modalities, as well as other non-operative therapies used in the treatment of HCC.

Keywords: Hepatocellular carcinoma (HCC); stereotactic body radiotherapy (SBRT); yttrium radioisotopes

Submitted Aug 26, 2015. Accepted for publication Sep 13, 2015.

doi: 10.21037/jgo.2015.10.04

View this article at: http://dx.doi.org/10.21037/jgo.2015.10.04 


\section{Introduction}

Hepatocellular carcinoma (HCC) accounts for the majority of primary liver malignant tumors and is responsible for more than half a million deaths annually worldwide (1). Chronic liver disease due to hepatitis $\mathrm{B}$ and $\mathrm{C}$ remains a leading risk factor for HCC globally and at least some degree of cirrhosis is seen in the majority of patients (2). Despite preventive measures with hepatitis B vaccine, HCC remains the third leading cause of cancer related mortality worldwide and is the fastest rising cause of cancer related death in the United States over the past two decades $(3,4)$.

Advances in surgical, medical and ablative therapies have improved long term survival rate and prognosis of HCC (5). Underlying liver dysfunction and pathological stage contribute to the survival rates and often preclude patients from curative options of either surgical resection or transplantation. Patients with non-resectable tumors can benefit from local tumor control through nonsurgical therapies such as external beam radiation therapy, radiofrequency ablation, or transarterial embolization. The delicate balance between curative radiation doses and occurrence of radiation-induced liver disease can limit the curative outcomes expected (6).

Historically, the use of external beam radiation to treat liver malignancies was limited due to low tolerance of whole liver irradiation (7). However, the advancements of stereotactic body radiation therapy has allowed for a highly focused targeted radiation to the tumor by delivering high dose of radiation precisely in single or few fractions while limiting toxicity to functional liver parenchyma (8). With improved techniques to account for the effects of respiratory motion on target volume (9-11) and advances in image guidance, stereotactic body radiotherapy (SBRT) is a noninvasive approach to definitive treatment of primary hepatic malignancies.

Selective internal radiotherapy (SIRT), also known as transarterial radioembolization (TARE) is an alternative locoregional strategy of delivering radioactive isotopes such as yttrium-90 to the tumor. The commercially available microspheres products are glass (TheraSpheres; MDS Nordion Ottawa, Ontario, Canada) and resin (Sir-Spheres; Sirtex Medical, Woburn, MA, USA). Retrospective review of ytrrium-90 infusions has shown that the efficacy of TARE depends on preserved target area blood flow in order to achieve high-energy beta-decay-induced free radical cell death (12).

To date, randomized comparison of SBRT to SIRT is lacking yet needed to define the role of radiation in treatment guidelines for HCC. Here we study a large population of patients solely with HCC. Using data from the Surveillance, Epidemiology, and End Results (SEER) registry of the National Cancer Institute from 2004 to 2011, we compare survival in HCC cancer patients who received highly conformal radiation with SBRT with those who received SIRT with SIR-Spheres. The purpose of this study is to determine whether SBRT or SIRT is associated with better outcomes in the treatment of HCC.

\section{Methods}

The SEER registry compiles cancer incidence, treatment, and survival data from 18 population-based cancer registries, covering more than $25 \%$ of the population of the United States. The SEER database was accessed to identify all patients diagnosed with HCC from 2004 (two years after FDA approval of SIR-spheres) until 2011 (most recent available data in the database). The variables obtained for each case include patient demographics (sex, race, age at presentation, year of diagnosis), tumor characteristics (size, histologic grade, surgical stage, extent of disease, nodal status of the disease, presence of distant metastases), AFP level, vital status, and treatment modalities (radiation sequence relative to surgery, type of surgery performed, external beam radiation, radioisotopes, and sequence of radiation with surgery).

\section{Patients}

The initial SEER query resulted in 50,386 cases of histologically diagnosed HCC between the years of 2001 and 2011. Cases with unknown stage $[8,171]$, unknown grade $[31,834]$, more than one primary tumor $[6,136]$, metastatic disease $[5,884]$, no radiation administered $[46,861]$, or unknown radiation modality $[1,379]$ were excluded. This generated a final cohort of 233 cases. Most of the cases with unknown stage were cases diagnosed prior to 2004, when a different coding scheme was used by the SEER database to describe the extent of disease. This coding scheme lacked the necessary information to fit patients into an AJCC stage group. Between 2002 and 2003 , only 3 cases of SIR-sphere use were reported and 71 cases of beam radiation treatment, however, the AFP level associated with those 3 cases was not reported. Any patients with surgery described as "Total hepatectomy and transplant" and "Hepatectomy NOS" were excluded. 
Patients with "preoperative radiation" were excluded. Thus the final cohort of 189 was included in this study from 2004 to 2011. There were 67 patients alive and 122 were expired at the time of analysis. Of the expired cases, liver specific disease caused 114 deaths, 8 deaths were attributed to other causes.

\section{Statistical analysis}

Chi-square test with exact $\mathrm{P}$ values based on Monte Carlo simulation and Wilcoxon's rank sum test were used to compare categorical variables, respectively. Overall survival (OS) is defined as the time (months) from the surgery to the last follow-up or death whichever occurs first (67 censored). The disease specific survival (DSS) is defined as the time (months) from the surgery to last follow-up or death whichever occurs first with death because of other reason treated as censored (75 censored). OS and DSS were compared between two radiation modalities using logrank test. The comparisons were further studied using multivariable Cox proportional hazard models to adjust for confounding factors which included age at diagnosis, sex, race, grade combined, stage combined, AFP level, type of surgery, tumor size. These factors were put into the regression model based on forward selection because of the small number of death events. Estimated hazard ratios (HRs) for each category versus the reference level and their $95 \%$ confidence intervals (CIs) were reported. For categorical variable, a $\mathrm{HR}>1$ means that category has more death risk or disease specific death risk than the reference category. For a continuous variable, a HR $>1$ means for every unit of increase in the variable, the death risk or the disease specific death risk also increases. Statistical analysis was performed using SAS 9.3 (SAS Institute, Inc., Cary, NC, USA) and statistical significance was set at 0.05 .

\section{Results}

Table 1 shows the descriptive statistics for categorical variables and continuous variables classified by radiation type. The table suggests that year of diagnosis, vital status and cause specific death were significantly associated with radiation type, reflecting the technological progress of these treatment modalities.

Figure 1 shows the Kaplan-Meier curves for OS and DSS, stratified by radiation type. The median OS time for patients treated with SBRT was 14 months (95\% CI: 10-18) compared to SIRT was 12 months (95\% CI: 9-17). The median DSS time for patients with SBRT was 14 months (95\% CI: 12-20) compared to SIRT was 14 months (95\% CI: 10-22).

Multivariable analysis showed that tumor size had a significant correlation with stage $(\mathrm{P}<0.0001)$. Further Cox $\mathrm{PH}$ models with forward selection were fit for variables radiation type, AFP level, radiation group with surgery, age at diagnosis, sex, race, grade and stage. The results are shown in Figures 2 and 3. After adjusting for other risk factors above, radiation type was not significantly associated with OS or disease-specific survival $(\mathrm{P}=0.1077$ for $\mathrm{OS}$ and $\mathrm{P}=0.0880$ for DSS). After adjusting for other risk factor, AFP level was significantly associated with OS and diseasespecific survival $(\mathrm{P}=0.0459$ for $\mathrm{OS}$ and $\mathrm{P}=0.0233$ for $\mathrm{DSS})$. Patients with elevated AFP level were associated with higher death risk and disease-specific death risk than AFP level within normal limits, regardless of the treatment modalities.

\section{Discussion}

In the present study, we aimed to compare the outcomes of treating unresectable cases of HCC with SBRT versus SIRT. The utilization of external beam radiation therapy for treatment of HCC has dramatically increased with the advent of stereotactic technique (SBRT). While SEER lacks detailed information on radiotherapeutic techniques, the recent conformal external beam radiation cases in SEER database are most likely in the form of SBRT. The retrospective clinical experience of Karolinska Hospital marked the first reported use of SBRT in treating patients with HCC (13). Since its publication in 1995, other retrospective studies have reported promising data suggesting that SBRT can serve as an alternative to surgery and bridge to liver transplantation $(14,15)$. Of note, the most recent and largest retrospective study by Sanuki et al. involving 185 patients with Child Pugh class A and B reported 1-year OS rate and local control of $95 \%$ and $99 \%$ respectively and $13 \%$ grade $>3$ toxicity (16). In addition, SBRT has a role in treatment of patients with tumor vascular thrombosis and advanced stage of HCC. The median survival for such patients is $2-4$ months without intervention (17). In contrast, with SBRT, a study of 56 HCC patients with tumor vascular thrombosis reported a median survival of 10.6 months and 1 year OS of $44 \%$ (18).

SBRT is currently indicated for patients with early stage disease and poor performance status precluding them from surgical resection or transplant, and those with portal vein invasion causing limited access for radioisotopes (19). Those 
Table 1 Descriptive statistics for categorical variables stratified by radiation type

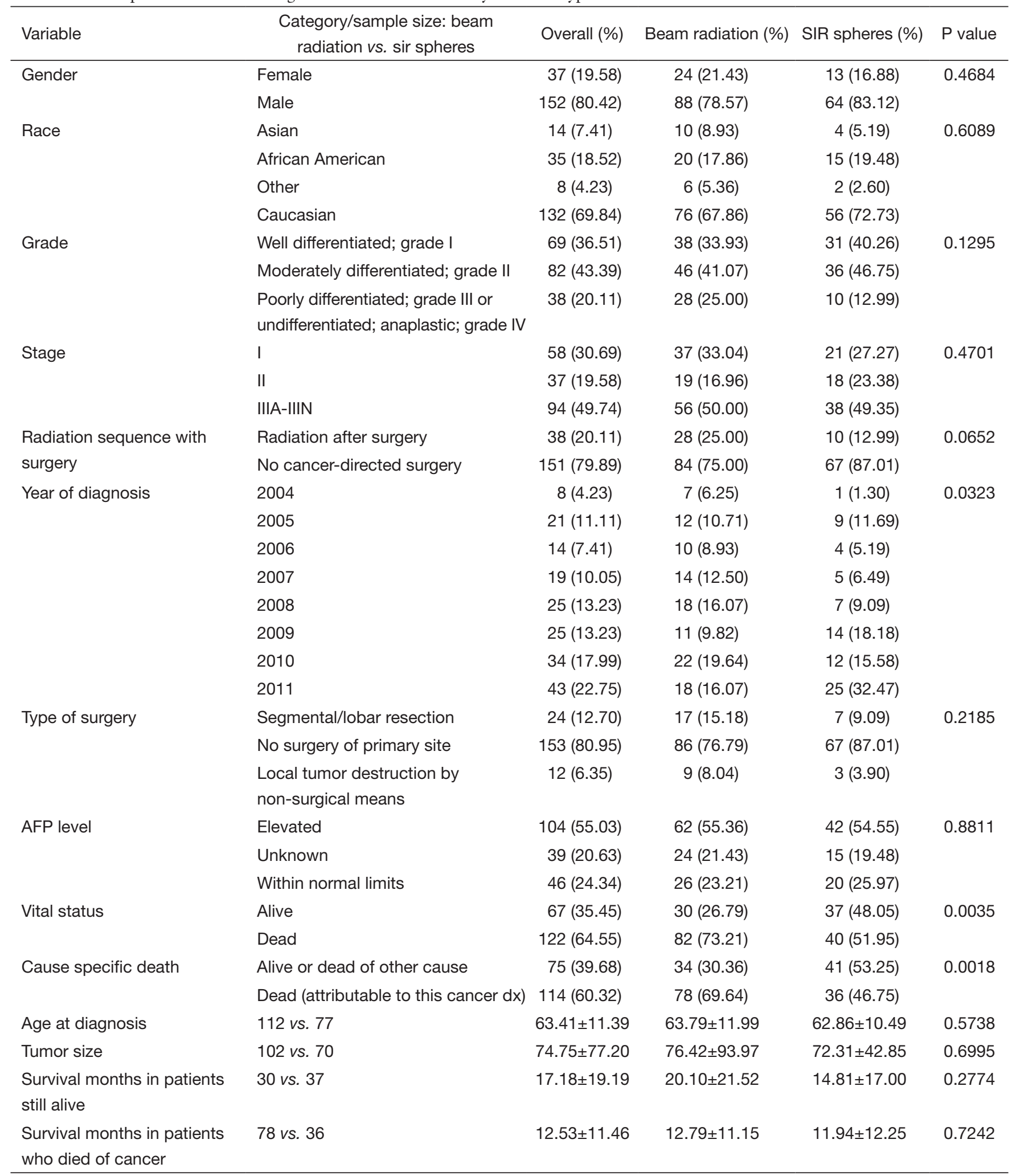

Table 1 (continued) 
Table 1 (continued)

\begin{tabular}{|c|c|c|c|c|c|}
\hline Variable & $\begin{array}{l}\text { Category/sample size: beam } \\
\text { radiation vs. sir spheres }\end{array}$ & Overall (\%) & Beam radiation (\%) & SIR spheres (\%) & $P$ value \\
\hline $\begin{array}{l}\text { Survival months in patients } \\
\text { who died of other causes }\end{array}$ & 4 vs. 4 & $12.88 \pm 13.12$ & $19.75 \pm 16.38$ & $6 \pm 2.71$ & 0.1886 \\
\hline $\begin{array}{l}\text { Survival months in all patients } \\
\text { who died }\end{array}$ & 82 vs. 40 & $12.55 \pm 11.51$ & $13.13 \pm 11.41$ & $11.35 \pm 11.77$ & 0.4297 \\
\hline
\end{tabular}
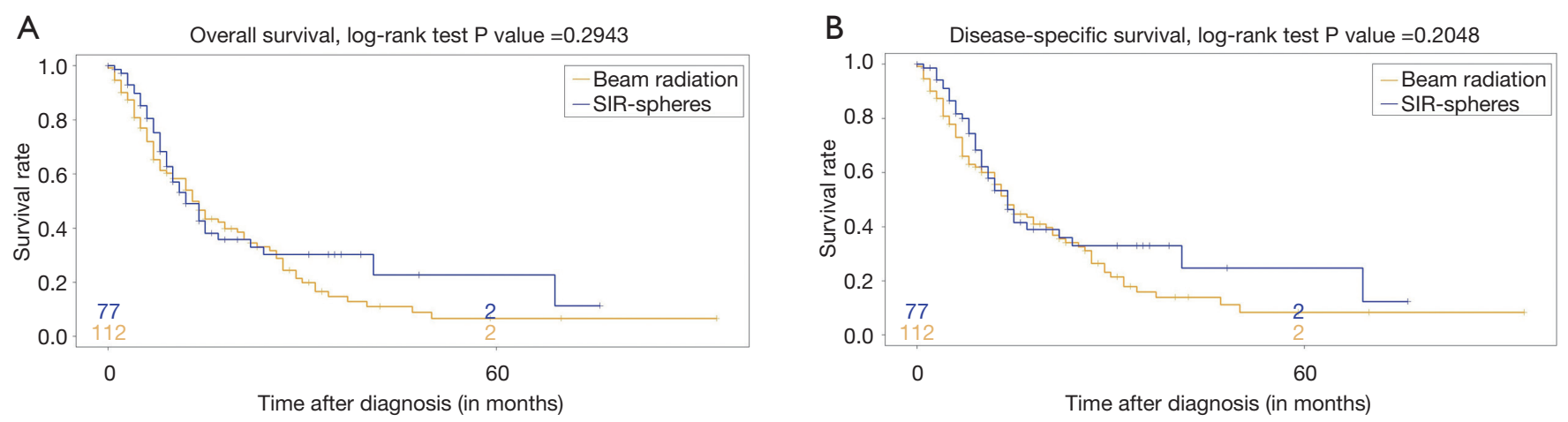

Figure 1 Kaplan-Meier curves for patients with hepatocellular carcinoma. (A) Overall survival of patients treated with SBRT versus SIRT; (B) disease-specific survival of patients treated with SBRT versus SIRT. SBRT, stereotactic body radiotherapy; SIRT, selective internal radiotherapy.

on transplant waiting lists can also benefit from SBRT as bridge therapy. Likewise, SIRT is indicated in patients with preserved liver function (serum total bilirubin $<2 \mathrm{mg} / \mathrm{dL}$ ), without ascites or hepatic encephalopathy and unresectable patients with portal vein thrombosis or poor-TACEcandidates (20). Patients with portal vein tumor thrombosis in the setting of HCC have been shown to benefit from TARE (21). The largest prospective study involving 291 patients with intermediate or advanced disease reported an overall time to progression of 7.9 months, difference in survival time between patients with Child-Pugh A or B disease (17.2 and 7.7 months respectively) and response rates between $42 \%$ and $57 \%$ based on WHO and EASL assessment criteria respectively (22).

Better quality of life in patients with unresectable HCC treated with SIRT compared to another form of TACE has been reported. SIRT has been shown to have fewer complications, decreased time to tumor progression and faster tumor response time compared to TACE $(23,24)$.
Thus as a form of brachytherapy, SIRT has a favorable outcome over other transarterial approaches to HCC treatment. While studies exist comparing TACE to TARE, randomized controlled trial comparing TARE to SBRT is lacking. In our study, after adjusting for risk factors, type of radiation was not significantly associated with OS or DSS. However, the AFP level was significantly associated with OS and disease-specific survival especially for those with elevated AFP level compared to normal limits.

The mean 5 -year survival rate of patients with HCC is known to depend on the presence or absence of elevated AFP, tumor size, resectability and symptoms. AFP positive tumors have a poorer prognosis as non-resectable cases have a mean survival of 5 months compared to AFP negative tumors with a mean survival of 10.5 months (25). Our findings of a higher death risk and disease-specific death risk for those with elevated AFP levels support this notion. In our study, the median DSS time was 14 months both for patients treated with SBRT (95\% CI: 12-20) and those 


\begin{tabular}{|c|c|c|c|}
\hline Variables & $\mathrm{P}$ value & & Hazard ratio $[95 \% \mathrm{Cl}]$ \\
\hline \multicolumn{4}{|l|}{ Radiation type } \\
\hline SIR-spheres vs. Beam radiation & 0.1077 & 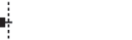 & $0.72[0.49,1.07]$ \\
\hline \multicolumn{4}{|l|}{ Gender } \\
\hline Female vs. male & 0.0922 & & $0.66[0.41,1.07]$ \\
\hline \multicolumn{4}{|l|}{ AFP level } \\
\hline Elevated vs. within normal limits & 0.0459 & - & $1.68[1.01,2.79]$ \\
\hline Unknown vs. within normal limits & 0.6934 & - & $1.13[0.62,2.05]$ \\
\hline \multicolumn{4}{|l|}{ Race } \\
\hline Asian vs. Caucasian & 0.4195 & 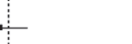 & $0.74[0.35,1.55]$ \\
\hline Other vs. Caucasian & 0.0275 & . & $-2.71[1.12,6.59]$ \\
\hline African American vs. Caucasian & 0.0344 & $=$ & $1.65[1.04,2.63]$ \\
\hline \multicolumn{4}{|l|}{ Grade } \\
\hline Moderately vs. poorly or undifferentiated & 0.8424 & - & $0.95[0.57,1.58]$ \\
\hline Well vs. poorly or undifferentiated & 0.0084 & & $0.48[0.28,0.83]$ \\
\hline \multicolumn{4}{|l|}{ Stage } \\
\hline | vs. IIIA-IIIN & 0.0262 & & $0.60[0.39,0.94]$ \\
\hline II vs. IIIA-IIIN & 0.0397 & & $0.54[0.30,0.97]$ \\
\hline \multicolumn{4}{|l|}{ Type of surgery } \\
\hline Local tumor destruction vs. no surgery & 0.0133 & & $0.38[0.18,0.82]$ \\
\hline \multirow[t]{4}{*}{ Surgical resection vs. no surgery } & 0.1123 & & $0.62[0.34,1.12]$ \\
\hline & ard ratio smaller & Hazard rat & bigger \\
\hline & roportional & $T$ & \\
\hline & 0.00 & 2.004 .00 & 8.00 \\
\hline
\end{tabular}

Figure 2 Forest plot for overall survival.

\begin{tabular}{|c|c|c|c|}
\hline Variables & $P$ value & & Hazard ratio $[95 \% \mathrm{Cl}]$ \\
\hline \multicolumn{4}{|l|}{ Radiation type } \\
\hline SIR-spheres vs. Beam radiation & 0.088 & (1) & $0.70[0.46,1.05]$ \\
\hline \multicolumn{4}{|l|}{ Gender } \\
\hline Female vs. male & 0.1688 & 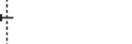 & $0.71[0.43,1.16]$ \\
\hline \multicolumn{4}{|l|}{ AFP level } \\
\hline Elevated vs. within normal limits & 0.0233 & & $1.86[1.09,3.19]$ \\
\hline Unknown vs. within normal limits & 0.5179 & - & $1.23[0.66,2.31]$ \\
\hline \multicolumn{4}{|l|}{ Race } \\
\hline Asian vs. Caucasian & 0.5531 & - & $0.80[0.37,1.69]$ \\
\hline Other vs. Caucasian & 0.0156 & & $-3.00[1.23,7.33]$ \\
\hline African American vs. Caucasian & 0.0642 & - & $1.59[0.97,2.59]$ \\
\hline \multicolumn{4}{|l|}{ Grade } \\
\hline Moderately vs. poorly or undifferentiated & 0.8655 & - & $0.96[0.57,1.61]$ \\
\hline Well vs. poorly or undifferentiated & 0.0062 & & $0.46[0.26,0.80]$ \\
\hline \multicolumn{4}{|l|}{ Stage } \\
\hline I vs. IIIA-IIIN & 0.019 & & $0.58[0.36,0.91]$ \\
\hline II vs. IIIA-IIIN & 0.0175 & & $0.47[0.25,0.88]$ \\
\hline \multicolumn{4}{|l|}{ Type of surgery } \\
\hline Local tumor destruction vs. no surgery & 0.0145 & & $0.39[0.18,0.83]$ \\
\hline Surgical resection vs. no surgery & 0.1173 & & $0.61[0.33,1.13]$ \\
\hline \multicolumn{2}{|c|}{ Hazard ratio smaller } & \multicolumn{2}{|c|}{ Hazard ratio bigger } \\
\hline \multicolumn{2}{|c|}{$\begin{array}{l}\text { P value is based on a multiple cox proportional } \\
\text { hazard regression model. }\end{array}$} & 1 & \\
\hline & 0.00 & 2.004 .00 & 8.00 \\
\hline
\end{tabular}

Figure 3 Forest plot for disease-specific survival. 
treated with SIRT (95\% CI: 10-22). When comparing solely radiation type, SBRT had a slightly longer survival time of 14 months (95\% CI: 10-18) compared to SIRT with 12 months (95\% CI: 9-17). The SEER database did not report the Child-Pugh Liver Cirrhosis score (i.e., A, $\mathrm{B}$ or $\mathrm{C}$ ), which could have impacted response rates and outcomes of liver directed radiation therapy. The database lacks information on patients pretreated with systemic chemotherapy, transcatheter arterial chemoembolization, radiofrequency ablation or percutaneous ethanol injection. Thus the effect of potential pretreatment with other noninvasive therapy is not accounted for in this retrospective study. SEER also lacks data on performance status, margin status, radiation dose and fields. However, published studies suggest a radiation dose-response relationship for local control and OS with SBRT (26).

\section{Conclusions}

In summary, this is the first retrospective review of large population-based data registry to show a similar overall and disease-specific survival benefit to treating cases of HCC with SBRT or SIRT. Our results, albeit retrospective, suggest similar outcomes for the two modalities in a large cohort of patients, validating the use of each but also indicating the need for further research to better define when one should be used over the other.

\section{Acknowledgements}

This work was supported by Stony Brook University Hospital.

\section{Footnote}

Conflicts of Interest: The authors have no conflicts of interest to declare.

\section{References}

1. World Health Organization Mortality Database. WHO Statistical Information System. 2008. Available online: http://www.who.int/whosis

2. Mittal S, El-Serag HB. Epidemiology of hepatocellular carcinoma: consider the population. J Clin Gastroenterol 2013;47 Suppl:S2-6.

3. Ferlay J, Shin HR, Bray F, et al. Estimates of worldwide burden of cancer in 2008: GLOBOCAN 2008. Int J Cancer 2010;127:2893-917.
4. Davila JA, El-Serag HB. The Rising Incidence of Hepatocellular Carcinoma in the United States: an Update. Gastroenterology 2012;142:S914.

5. Klein J, Dawson LA. Hepatocellular carcinoma radiation therapy: review of evidence and future opportunities. Int J Radiat Oncol Biol Phys 2013;87:22-32.

6. Guha C, Kavanagh BD. Hepatic radiation toxicity: avoidance and amelioration. Semin Radiat Oncol 2011;21:256-63.

7. Ingold JA, Reed GB, Kaplan HS, et al. Radiation hepatitis. Am J Roentgenol Radium Ther Nucl Med 1965;93:200-8.

8. Potters L, Kavanagh B, Galvin JM, et al. American Society for Therapeutic Radiology and Oncology (ASTRO) and American College of Radiology (ACR) practice guideline for the performance of stereotactic body radiation therapy. Int J Radiat Oncol Biol Phys 2010;76:326-32.

9. Eccles CL, Dawson LA, Moseley JL, et al. Interfraction liver shape variability and impact on GTV position during liver stereotactic radiotherapy using abdominal compression. Int J Radiat Oncol Biol Phys 2011;80:938-46.

10. Case RB, Sonke JJ, Moseley DJ, et al. Inter- and intrafraction variability in liver position in non-breathhold stereotactic body radiotherapy. Int J Radiat Oncol Biol Phys 2009;75:302-8.

11. Wong JW, Sharpe MB, Jaffray DA, et al. The use of active breathing control $(\mathrm{ABC})$ to reduce margin for breathing motion. Int J Radiat Oncol Biol Phys 1999;44:911-9.

12. Salem R, Thurston KG. Radioembolization with 90Yttrium microspheres: a state-of-the-art brachytherapy treatment for primary and secondary liver malignancies. Part 1: Technical and methodologic considerations. J Vasc Interv Radiol 2006;17:1251-78.

13. Blomgren H, Lax I, Näslund I, et al. Stereotactic high dose fraction radiation therapy of extracranial tumors using an accelerator. Clinical experience of the first thirty-one patients. Acta Oncol 1995;34:861-70.

14. Tse RV, Hawkins M, Lockwood G, et al. Phase I study of individualized stereotactic body radiotherapy for hepatocellular carcinoma and intrahepatic cholangiocarcinoma. J Clin Oncol 2008;26:657-64.

15. O'Connor JK, Trotter J, Davis GL, et al. Long-term outcomes of stereotactic body radiation therapy in the treatment of hepatocellular cancer as a bridge to transplantation. Liver Transpl 2012;18:949-54.

16. Sanuki N, Takeda A, Oku Y, et al. Stereotactic body radiotherapy for small hepatocellular carcinoma: a retrospective outcome analysis in 185 patients. Acta Oncol 2014;53:399-404. 
17. Llovet JM, Bustamante J, Castells A, et al. Natural history of untreated nonsurgical hepatocellular carcinoma: rationale for the design and evaluation of therapeutic trials. Hepatology 1999;29:62-7.

18. Bujold A, Massey CA, Kim JJ, et al. Sequential phase I and II trials of stereotactic body radiotherapy for locally advanced hepatocellular carcinoma. J Clin Oncol 2013;31:1631-9.

19. Lo SS, Dawson LA, Kim EY, et al. Stereotactic body radiation therapy for hepatocellular carcinoma. Discov Med 2010;9:404-10.

20. Raoul JL, Sangro B, Forner A, et al. Evolving strategies for the management of intermediate-stage hepatocellular carcinoma: available evidence and expert opinion on the use of transarterial chemoembolization. Cancer Treat Rev 2011;37:212-20.

21. Lau WY, Sangro B, Chen PJ, et al. Treatment for hepatocellular carcinoma with portal vein tumor thrombosis: the emerging role for radioembolization using yttrium-90. Oncology 2013;84:311-8.

22. Salem R, Lewandowski RJ, Mulcahy MF, et al.

Cite this article as: Oladeru OT, Miccio JA, Yang J, Xue Y, Ryu S, Stessin AM. Conformal external beam radiation or selective internal radiation therapy - a comparison of treatment outcomes for hepatocellular carcinoma. J Gastrointest Oncol 2016;7(3):433-440. doi: 10.21037/jgo.2015.10.04
Radioembolization for hepatocellular carcinoma using Yttrium-90 microspheres: a comprehensive report of longterm outcomes. Gastroenterology 2010;138:52-64.

23. Kooby DA, Egnatashvili V, Srinivasan S, et al. Comparison of yttrium-90 radioembolization and transcatheter arterial chemoembolization for the treatment of unresectable hepatocellular carcinoma. J Vasc Interv Radiol 2010;21:224-30.

24. Salem R, Lewandowski RJ, Kulik L, et al. Radioembolization results in longer time-to-progression and reduced toxicity compared with chemoembolization in patients with hepatocellular carcinoma. Gastroenterology 2011;140:497-507.e2.

25. Hollinger FB, Liang TJ. Hepatitis B Virus. In: Knipe DM, Howley PM, Griffin DE, et al, editors. Fields Virology, 4th ed. Philadelphia: Lippincott Williams \& Wilkins, 2001:2971-3036.

26. Jang WI, Kim MS, Bae SH, et al. High-dose stereotactic body radiotherapy correlates increased local control and overall survival in patients with inoperable hepatocellular carcinoma. Radiat Oncol 2013;8:250. 